\title{
AVALIAÇÃO DO EFEITO GENOTÓXICO DA MEMBRANA POLIHIDROXIBUTIRATO/NORBIXINA/ETILENOGLICOL
}

\author{
Adrielle Martins Monteiro Alves ${ }^{1}$ \\ Rayssilane Cardoso de Sousa ${ }^{2}$ \\ Thaís Márjore Pereira de Carvalho ${ }^{3}$ \\ Luiz Fernando Meneses de Carvalho ${ }^{4}$ \\ Antônio Luiz Martins Maia Filho ${ }^{5}$ \\ Samylla Miranda Monte ${ }^{6}$ \\ Danniel Cabral Leão Ferreira ${ }^{7}$ \\ Kethyma Morreira Fonseca ${ }^{8}$ \\ Deuzuita Santos Oliveira ${ }^{9}$ \\ Vicente Galber Freitas Viana ${ }^{10}$
}

Resumo: Biomateriais são substâncias de origem sintética ou natural que substituem e/ou estimulam sistemas biológicos que deixaram de ter suas funções, como exemplo a restauração de funções teciduais. Tendo em vista o potencial antioxidante da norbixina (carotenoide dicarboxílico encontrado na Bixa orellana L.) e de biodegradabilidade e biocompatibilidade do polihidroxibutirato (PHB) (polímero natural sintetizado a partir de bactérias), objetivou-se preparar uma membrana a partir destes dois constituintes com a adição do reagente etilenoglicol (PHB/norbixina/etilenoglicol) para avaliar seus efeitos genotóxico por meio do teste de micronúcleo. Para este estudo, 15 ratos foram divididos em 3 grupos: $A$ - a membrana foi introduzida no peritônio dos animais através de uma laparotomia; $B$ - apenas uma laparotomia com água destilada; $C$ injeção de ciclofosfamida em dose única de $50 \mathrm{mg} / \mathrm{kg}$ por via intraperitoneal. Foi coletado material de medula óssea de cada rato para realizar o teste de micronúcleo. Em conclusão, o teste de micronúcleo sugere que a membrana não é genotóxica.

Palavras-chave: Genotoxicidade; Mutagenicidade; Biomaterais; Polihidroxibutirato; Norbixina.

\footnotetext{
${ }^{1}$ Mestranda em Engenharia Biomédica/UNIVAP, Brasil. E-mail: adriellemonteiro@hotmail.com.

2 Mestranda em Engenharia de Materiais/IFPI, Brasil. E-mail: rayssilane14@hotmail.com.

${ }^{3}$ Mestranda em Engenharia de Materiais/IFPI, Brasil. E-mail: thaismarjore.pc@gmail.com.

${ }^{4}$ Doutorado em Ciências e Engenharia dos Materiais/UFRN, Brasil. E-mail: luizfmc01@gmail.com.

${ }^{5}$ Doutorado em Engenharia Biomédica/UNIVAP, Brasil. E-mail: almmaiaf@gmail.com.

${ }^{6}$ Doutorado em Ciências Morfológicas/UFRJ, Brasil. E-mail: samylla_monte@hotmail.com.

${ }^{7}$ Medicina Veterinária/UFPI, Brasil. E-mail: dannielclf@hotmail.com.

${ }^{8}$ Fisioterapia/Uespi, Brasil. E-mail: ketyma_mf@hotmail.com.

9 Doutorado em Engenharia Mecânica/USP, Brasil. E-mail: deuzuitasantos@globo.com.

${ }^{10}$ Doutorado em Ciências (Física Aplicada)/USP, Brasil. E-mail: galber@ifpi.edu.br.
} 